\title{
Modelling and Performance Measure of a Perinatal Network Centre in the United Kingdom
}

\author{
Md Asaduzzaman and Thierry J. Chaussalet \\ Health and Social Care Modelling Group (HSCMG) \\ School of Informatics, University of Westminster \\ 115, New Cavendish Street, London W1W 6UW \\ United Kingdom \\ asaduzm@wmin.ac.uk, chausst@wmin.ac.uk
}

\begin{abstract}
The main aim of this paper is to model the neonatal unit of a perinatal network centre using the general framework of a loss network model and to estimate some performance measures. A special case of the class of model has been applied for capacity planning to the perinatal network centre of a neonatal network in the United Kingdom. Using the data supplied from the perinatal network centre about admission process, length of stay $(L o S)$ and discharge pattern of the babies, the loss network model is applied to estimate the admission refusal probability in the system under steady-state conditions. Results are derived for different arrival patterns and different combinations of cots at all levels of care of the neonatal unit. This approach can be useful to select the optimal combination of cots for any given acceptance rate of arrival to the neonatal unit.
\end{abstract}

\section{Introduction}

Every neonatal unit in the United Kingdom (UK) aims to offer high quality care for the vulnerable babies in the society. These neonatal units in the UK are organised into neonatal networks where a network consists of several units. Most of these neonatal units are regularly unable to meet the demand [10]. The premature baby charity BLISS reported that about $78 \%$ of all neonatal units had to close to new admissions at least once in the last six months [2]. This problem is exacerbated by several reasons. First, the percentage of refusal of admission from all neonatal units in UK is increasing. Secondly, these refusals of admission create risk and expensive transfer of sick babies. Thirdly, cots in neonatal units are very expensive, so management wants to keep these resources highly utilised. Consequently, the number of cots is inadequate. A perinatal network centre has a neonatal unit which consists of three units providing different levels of care: intensive care unit (ICU), high dependency unit (HDU)and special care unit (SCU). It is also known as the lead centre of the neonatal network and plays a vital role by providing the highest level of treatment and managing transfers of babies to other units for the most vulnerable babies. Many queueing network models and simulation models have been developed in health care research to model patients flow, manage capacity etc. $[6][9][3][1][5][8][11]$. In terms of modelling neonatal units, few articles have been found in the literature. However, most of the previous methods used both in neonatal care and health care in general did not capture the current issues of neonatal units. In a neonatal unit no queue is allowed to form. If a neonatal unit of a network runs out of capacity, babies who arrive to be admitted, are immediately transferred to another unit where places are available. Therefore, it is necessary to develop a suitable model to capture the flow of the babies in the units and measure the key performances. This paper provides a mathematical model for a neonatal unit with different levels of care through a loss network approach. The model contains a detailed description of the classes of babies admitted to a neonatal unit, and of solutions to accommodate cot shortages. Typical solutions in case of cot shortage are: transferring a baby to another unit/region; transferring to transitional care unit or releasing another baby earlier. But these alternatives have serious drawbacks, and also depend on the severity of sickness of the babies. The activity of a perinatal network centre or a neonatal unit has major similarities with loss network models developed for circuit switched telephone systems. For such systems, loss network model can be used to approximate the fraction of blocked telephone calls. In this paper, we propose such a generalised framework of a loss network model to capture patient flow and estimate performance measures of a network of units in equilibrium state. 
The University College London Hospital (UCLH) perinatal centre of the North Central London Perinatal Network (NCLPN) is one of the busiest perinatal network centres across the country. The data for UCLH perinatal network centre have been used for a particular case of a loss network model. The model derives exact loss probability of the fraction of refused babies. The modelling approach allows us to determine the number of cots for any given acceptance rate of admission in all units of a perinatal network centre. This approach will be useful for future service improvement in perinatal centres of neonatal networks in the UK or in other countries where neonatal care is organised in a similar fashion.

\section{Data and preliminary results}

The data used for this study concerns the UCLH perinatal network centre. Data collection was done through the South England Neonatal Database (SEND) and NCLPN Transfer Audit. The data provide comprehensive and accurate information about each baby's stay in the ICU/HDU and SCU, including dates and times of arrival/discharge, source and reason of admission, birth weight and gestational weeks, admission refusal time and date. All 1002 babies admitted to the UCLH neonatal unit in the year 2006 were considered eligible for the study. The interarrival time and length of stay are calculated in days from the recorded dates of arrival and discharge. Of the 1002 babies who were admitted to UCLH in $2006,31 \%$ were admitted to the intensive care and high dependency units and the remaining were admitted to the special care unit. Summary statistics of interarrival time and length of stay $(\mathrm{LoS})$ are presented in Table 1. Fig. 1 shows the interarrival and LoS distribution for both units.

Table 1. Summary statistics of the interarrival times and lengths of stay, in days of babies in UCLH

\begin{tabular}{lccc}
\hline Summary statistics & No. of babies & Mean & SD \\
\hline ICU/HDU & & & \\
Interarrivals (in days) & 443 & 0.82 & 0.997 \\
LoS (in days) & 308 & 14.10 & 20.33 \\
\hline SCU & & & \\
Interarrivals (in days) & 905 & 0.40 & 0.57 \\
LoS (in days) & 884 & 7.76 & 7.63 \\
\hline
\end{tabular}
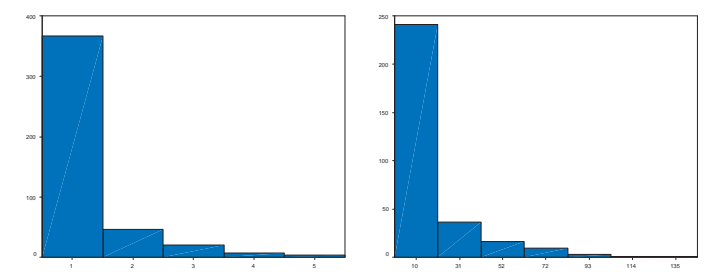

(a) Interarrival time at ICU and (b) Length of stay at ICU and HDU. HDU.

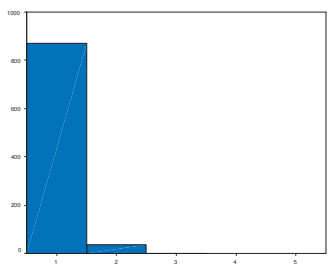

(c) Interarrival time at SCU.

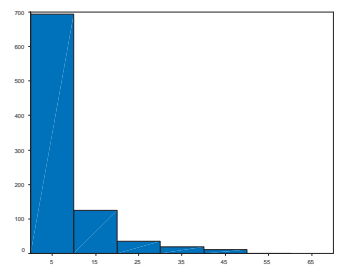

(d) Length of stay at SCU.
Figure 1. Histogram of interarrival time and length of stay for all units.

\section{Proposed model framework}

Babies arriving at a perinatal network centre are of three classes, level I or intensive care, high dependency or level II care and special or level III care. Class depends on the severity of sickness of a baby. In principle, a baby entering an intensive care unit is normally transferred to high dependency unit depending upon improvement and then transferred to special care from where he/she is allowed to go home/normal ward. But since the number of cots in all three levels is small and the cots in intensive and high dependency care are in high demand, babies coming from outside the network are normally transferred from intensive and high dependency care back to the units from where they come. Therefore, exit from any unit is still possible. An important characteristic of the unit is- it does not allow waiting for admission. In such cases, a baby is transferred to another neonatal unit.

\subsection{A generalised loss network model}

In Fig. 2 we schematically depict the patient flows for a general loss network model for a perinatal network centre. The network consists of $\mathbf{J}$ units, labelled $1,2,, J$. Unit $j$ has $C_{j}$ cots. Let $\mathcal{K}$ be the set of classes of babies admitted to a perinatal network centre, with $|\mathcal{K}|=K$. The number of units and the number of classes of babies admitted to a perinatal network centre are usually the same and typically three (intensive, high dependency and special). A baby of class- $k$ requires $A_{j k}$ units of cots on unit $j$, with $A_{j k}$ a non- 
negative integer. $A_{j k}=0$ implies class- $k$ babies do not go to unit $j$.

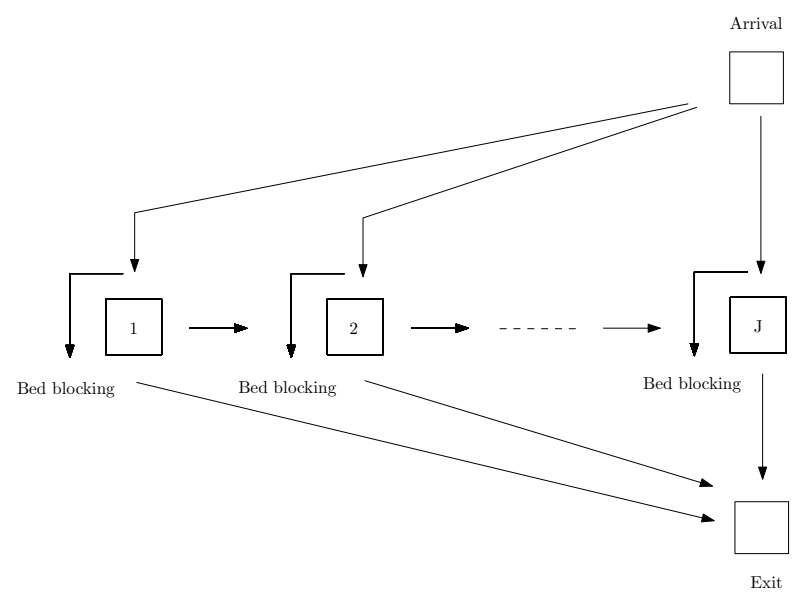

Figure 2. A general loss network model for a perinatal network centre.

Since each unit in our loss network framework provides a single type of service, the $A_{j k} \in 0,1$. Let $\mathcal{I}_{k}=\{j$ : $\left.A_{j k}>0\right\}$ be the set of units (the route) admitting the class$k$ babies, with $|\mathcal{I}|=J_{k}$. Let $|\mathcal{K}|=\left\{k: A_{j k}>0\right\}$ be the set of classes of babies admitted to unit $j$, with $\left|\mathcal{K}_{j}\right|=K_{j}$.

Babies arrive according to a Poisson process with rate $\lambda_{k}$ for a particular class, independent of arrival processes for the other classes. A new baby of class- $k$ is blocked and transferred to another unit if, in any unit $j \in \mathcal{I}_{k}$, there are fewer than $A_{j k}$ free cots. Otherwise, the baby is admitted and simultaneously uses $A_{j k}$ units of cots from every unit $j \in \mathcal{I}_{k}$ for an arbitrarily distributed length of stay with mean $1 / \mu_{k}$. This length of stay is assumed independent of the arrival process and the length of stay of the babies admitted into other units.

We use $n=\left(n_{k}: k \in \mathcal{K}\right)^{T}$ to denote the system state of the network, where $n_{k}$ is the number of class- $k$ babies currently in the network. Let $\mathbf{C}=\left(C_{1}, C_{2}, \ldots, C_{J}\right)^{T}$ be the vector of capacities for the $J$ units and $A=\left(A_{j k}: j=\right.$ $1,2, \ldots, J ; k \in \mathcal{K})$ be the routing matrix. Hence, the set of all possible system states is denoted by

$$
\boldsymbol{\Omega}=\left\{n \in \mathbb{Z}_{K}^{+}: \sum_{k} A_{j k} n_{k} \leq C_{j}, 1 \leq j \leq J\right\}
$$

The steady-state probability of the network being in state $n \in \Omega$ is given by (cf. [7][12])

$$
\pi(n)=\frac{1}{G} \prod_{k \in \mathcal{K}} \frac{\rho_{k}^{n_{k}}}{n_{k} !}
$$

where

$$
G=\sum_{n \in \Omega} \prod_{k \in \mathcal{K}} \frac{\rho_{k}^{n_{k}}}{n_{k} !}
$$

is called the normalisation constant and $\rho_{k}=\lambda_{k} / \mu_{k}$ is the offered load of class- $k$ babies. Equation (2) is often referred to as the product-form solution of a loss network. The insensitivity property [12] ensures that the product-form solution holds for general (not necessarily exponential) service time distributions with finite means. Let $B_{k}$ be the steady-state probability that a new class- $k$ baby will be refused admission i.e. blocked by the network. It can be then calculated as follows

$$
\begin{aligned}
B_{k} & =1-\sum_{n \in \Omega\left(C-A I_{k}\right)} \pi(n) \\
& =1-\frac{G\left(C-A I_{k}\right)}{G(C)}
\end{aligned}
$$

where $I_{k}$ is the unit vector with the $k$ th element being 1 and the other elements being 0 . However, for most practical networks, direct calculation of the normalization constant is difficult, because it requires summing over all the states in $\Omega$. Several approximate techniques were proposed to compute the normalization constant and the blocking probabilities such as reduced load approximation (RLA) [7][4] and Monte Carlo method [13].

\subsection{A loss network model for the UCLH perinatal network centre}

We introduce here a special case of a general loss network model which is applied to UCLH neonatal unit, the perinatal network centre of the neonatal network in North Central London. UCLH, intensive and high dependency care units have been merged for the purpose of better utilisation of cots. Therefore, the model for UCLH has two units: an intensive-high dependency unit and a special care unit. Babies cared at the intensive-high dependency unit may go to the special care unit and vice versa in some cases. However, in such cases we assume babies get no priority to be admitted to the corresponding unit. They are simply treated as new arrivals. In fact, this assumption is realistic, and allows us to consider each unit independently and consequently to reduce computational complexities. If no cot is available in any of the units upon arrival, the baby is sent to another unit. Babies can be discharged from any of the units. Fig. 3 shows the flow of patients at UCLH neonatal unit. From a mathematical perspective, the behaviour of the units in a neonatal unit closely resembles that of a circuit switched telephone system. A call generated is blocked when all circuits are occupied and eventually lost. But in the neonatal case we assume the baby is transferred elsewhere. The issue of bed blocking has received considerable 
attention for all the neonatal units in UK recently. In the simplest case of one telephone switch with one incoming flow and $c$ circuits, the system is referred to as the Erlang loss system, and the blocking probability can be computed using the famous Erlang loss formula [12]

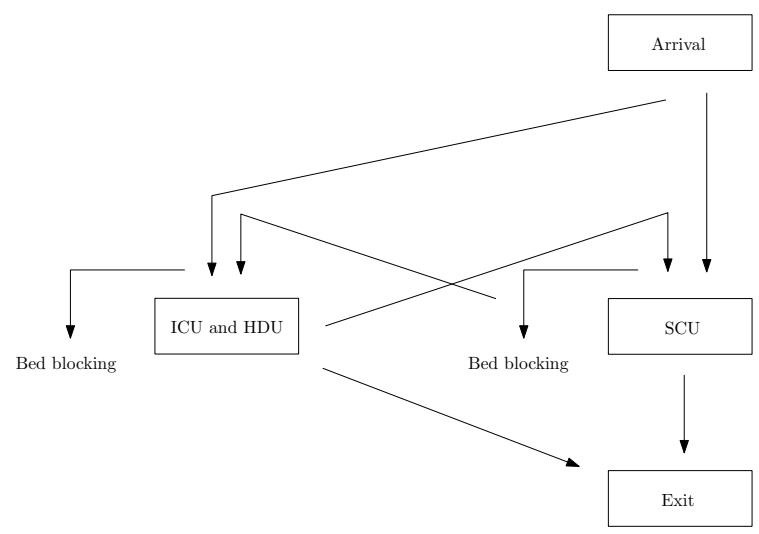

Figure 3. A loss network model for UCLH perinatal network centre.

$$
B\left(c_{j}, \rho_{j}\right)=\frac{\rho_{j}^{c_{j}} / c_{j} !}{\sum_{k=0}^{J} \rho_{k}^{n_{k}} / n_{k} !}
$$

where $\rho=\lambda / \mu$ is the load with $\lambda$ the arrival rate and $1 / \mu$ the mean length of stay. To consider a more realistic model we assume that blocked patients overflow into a common unit and this allows us to approximate these blocking probabilities in this model using the Equivalent Random Method (ERM) introduced by Wilkinson [14]. The idea behind the classical ERM is to replace several multi-server units by one equivalent random unit that generates the same expectation and variance of the overflow as in the original system. Let us consider a network of $J$ units each having several cots. Using the ERM, the mean and variance of the number of babies in the overflow from unit $j=1,2, \ldots, J$ with load $\rho_{j}=\lambda_{j} / \mu_{j}$ and capacity $c_{i}$ are

$$
\begin{gathered}
E_{j}=\rho_{j} B\left(c_{j}, \rho_{j}\right) \\
V_{j}=E_{j}\left(1-E_{j}+\frac{\rho_{j}}{c_{j}+1+E_{j}-\rho_{j}}\right)
\end{gathered}
$$

The mean and variance of the total number of patients in the overflow, assuming that the overflow buffer has an infinite capacity, is

$$
E=\sum_{j=1}^{J} E_{j}, V=\sum_{j=1}^{J} V_{j}
$$

We perform the ERM to calculate the mean numbers of patients blocked in the system and overflowed which represents the number of babies transferred to other units.

\section{Numerical results}

The main aim of this article is to investigate how the number of cots and the arrival process affect the rejection of admission. To investigate this through the loss network model, we derive the rejection probabilities in terms of the number of cots and also in terms of the arrival rate for both units.

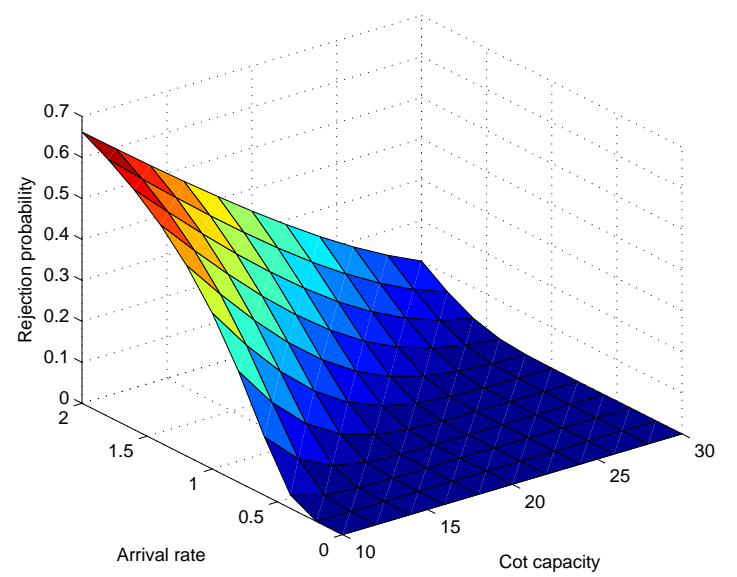

Figure 4. Rejection probability at ICU-HDU in terms of arrival rate and number of cots.

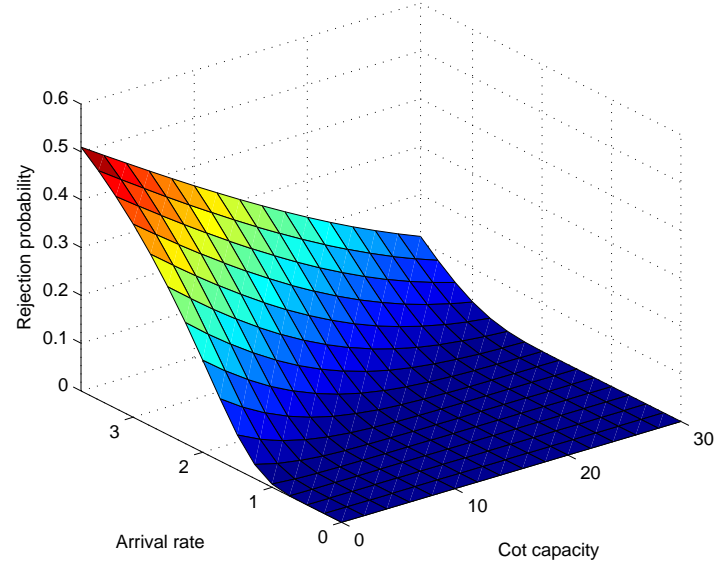

Figure 5. Rejection probability at SCU in terms of arrival rate and number of cots.

The results obtained by the loss network model are presented in Fig. 4 and Fig. 5. For the ICU-HDU, admission rejection probability increases with the arrival rate and de- 


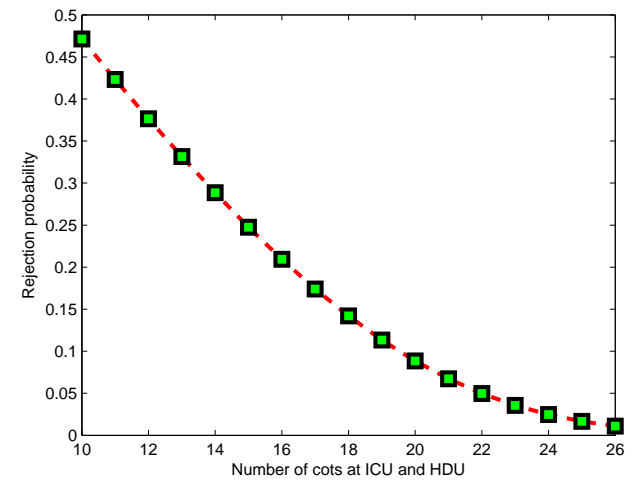

(a) Rejection probability at ICU-HDU.

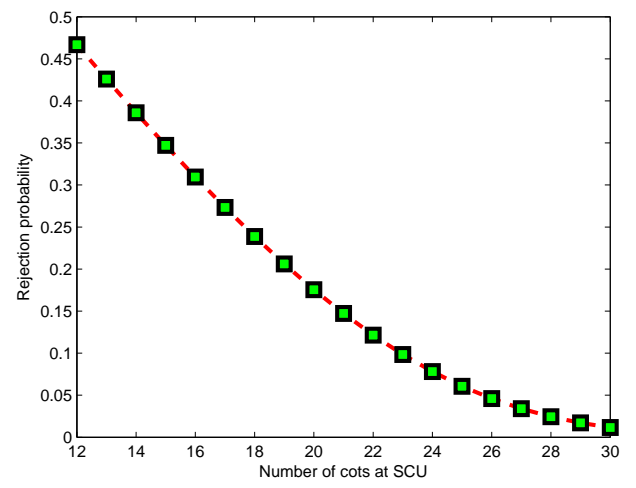

(b) Rejection probability at SCU.

Figure 6. Rejection probability for both units in terms of number of cots.

creases with the number of cots for the intensive-high dependency unit. This result is particularly useful to select the number of cots for a certain level of rejection at any unit. With the existing arrival pattern, the choice of number of beds can be easily obtained using this model. Fig. 6 shows the rejection probability in terms of the number of cots at each care unit for the existing arrival pattern.

At UCLH, there were 12 intensive and high dependency care cots, 15 special care cots and 8 transitional care cots in 2006. The special care cots were sometimes used as intensive and high dependency care cots depending upon availability and circumstance and the transitional cots were sometimes used as replacement of special care cots. From the cot occupancy reported in the annual report 2006 of North Central London Perinatal Network (NCLPN), it can be observed that on average one special cot has been used for intensive and high dependency care and all 8 transitional care cots have been used for special and transitional care. With the existing arrival pattern, a baby arriving at UCLH ICU-HDU has a probability 0.377 of being refused admis-
Table 2. Rejection probability at UCLH

\begin{tabular}{lcc}
\hline Unit & No. of cots & Calculated rejection prob. \\
\hline ICU/HDU & & \\
& 12 & 0.377 \\
& 13 & 0.332 \\
\hline SCU & 15 & 0.347 \\
& 23 & 0.098 \\
\hline
\end{tabular}

sion due to the lack of capacity. The corresponding probability for the special care unit is 0.347 . However, when we account for the one special care cots at ICU-HDU, the rejection probability becomes 0.332 , which exactly matches with the observed value. The corresponding rejection probability for special care unit becomes 0.098 when we account the extra 8 transitional care cots, which is also close to the observed value. The equivalent random method can be used to calculate the mean numbers of babies blocked in a particular unit and then transferred to another unit. Using ERM the mean number of babies rejected from the intensive-high dependency unit is 6 with variance 11 and from the special care unit it is 2 with variance 4 .

\section{Conclusion and further research}

All recent studies about neonatal care networks in the UK suggest that capacity is now a burning issue. It is claimed that capacity needs to be expanded immediately for most of the units in the UK. But the cost involved in running neonatal cots is so high that expanding capacity can only be limited. A cot itself in neonatal units is also very expensive. Staffing associated with cots, particularly in level II and level III is very high. Transfer of neonates within or between units is also a big issue since it involves risk and cost. Quality of care is another issue. The proposed loss model incorporates the property of no queue in the units and gives us a very quick idea about the capacity required for a prespecified performance level. The loss probabilities calculated by the model are close to those derived directly from the data. The other main advantage of this model is its simplicity. Hence, the model can be used as a capacity planning tool for a perinatal network centre of a neonatal network for a given level of rejection. However, a more appropriate approach might be to model explicitly the transitional care unit within the system which is currently being investigated.

\section{Acknowledgement}

The authors acknowledge Nicola J. Robertson, Department of Obstetrics and Gynaecology, University College London 
and Daniel Wood, Maternity and Neonatal Service, University College London Hospital for their help regarding data gathering and anonymisation.

\section{References}

[1] S. W. M. Au-Yeung, P. G. Harrison, and W. J. Knottenbelt. A Queueing Network Model of Patient Flow in an Accident and Emergency Department. In 20th Annual European and Simulation Modelling Conference, 2006.

[2] BLISS. Neonatal Services -are they improving? BLISS The Premature Baby Charity, 2005.

[3] T. J. Chaussalet, H. Xie, and P. Millard. A closed queueing approach to the analysis of patient flow in health care systems. Methods of Information in Medicine, 5:492-497, 2006.

[4] S. P. Chung and K. W. Ross. Reduced load approximation for multirate loss networks. IEEE Transactions on Communications, 41:1222-1231, 1993.

[5] E. El-Darzi, C. Vasilakis, T. Chaussalet, and P. Millard. A simulation modelling approach to evaluating length of stay, occupancy, emptiness and bed blocking in a hospital geriatric department. Health Care Management Science, 1(2):143-149, 1998.

[6] J. D. Griffiths and N. Price-Lloyds. A queueing model of activities in an intensive care unit. IMA Journal of Management Mathematics, 17(3):277-288, 2006.

[7] F. P. Kelly. Loss network. Annals of Applied Probability, 1(3):319-378, 1991.

[8] S. Kim, I. Horowitz, K. K. Young, and T. A. Buckley. Analysis of capacity management of the intensive care unit in a hospital. European Journal of Operational Research, 115(1):36-46, 1999.

[9] N. Koizumi, E. Kuno, and T. E. Smith. Modelling patient flows using a queuing network with blocking. Health Care Management Science, 8(1):49-60, 2005.

[10] J. Parmanum, D. Field, J. Rennie, and P. Steer. National census of availability of neonatal intensive care. British Medical Journal, 321(7263):727-729, 2000.

[11] J. Ridge, S. Jones, M. Nielsen, and A. Shahani. Capacity planning for intensive care units. European Journal of Operational Research, 105(2):346-355, 1998.

[12] K. Ross. Multiservice Loss Models for Broadband Telecommunication Networks. Springer-Verlag, 1995.

[13] K. Ross and J.Wang. Monte carlo summation applied to product-form loss networks. Probability in the Engineering and Informational Sciences, 6:323-348, 1992.

[14] R. Wilkinson. Theories for toll traffic engineering in the usa. The Bell System Technical Journal, 40:421-514, 1956. 